\title{
Selective Functionalization Blended with Scaffold Conductivity in Graphene Acid Promotes $\mathrm{H}_{2} \mathrm{O}_{2}$ Electrochemical Sensing
}

\author{
Anna Lenarda, ${ }^{\dagger}$ Aristides Bakandritsos, ${ }^{\S}$ Manuela Bevilacqua, ${ }^{\ddagger}$ Claudio Tavagnacco, ${ }^{\dagger}$
}

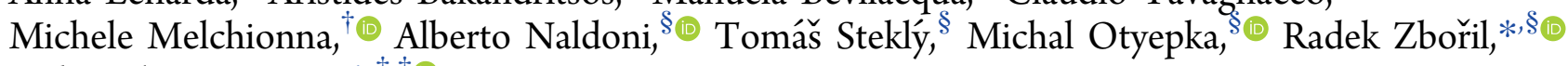
and Paolo Fornasiero $*,+\notin \bullet$

\footnotetext{
${ }^{\dagger}$ Department of Chemical and Pharmaceutical Sciences, INSTM and ${ }^{\ddagger}$ ICCOM-CNR, University of Trieste, Via L. Giorgieri 1, 34127 Trieste, Italy

${ }^{\S}$ Regional Centre of Advanced Technologies and Materials and Department of Physical Chemistry, Faculty of Science, Palacký University Olomouc, Šlechtitelu 27, 78371 Olomouc, Czech Republic
}

Supporting Information

ABSTRACT: The widespread industrial use of $\mathrm{H}_{2} \mathrm{O}_{2}$ has provoked great interest in the development of new and more efficient materials for its detection. Enzymatic electrochemical sensors have drawn particular attention, primarily because of their excellent selectivity. However, their high cost, instability, complex immobilization, and inherent tendency toward denaturation of the enzyme significantly limit their practical usefulness. Inspired by the powerful proton-catalyzed $\mathrm{H}_{2} \mathrm{O}_{2}$ reduction mechanism of peroxidases, we have developed a well-defined and densely functionalized carboxylic graphene derivative (graphene acid, GA) that serves as a proton source and conductive electrode for binding and detecting $\mathrm{H}_{2} \mathrm{O}_{2}$. An unprecedented $\mathrm{H}_{2} \mathrm{O}_{2}$ sensitivity of $525 \mu \mathrm{A} \mathrm{cm} \mathrm{cm}^{-2} \mathrm{mM}^{-1}$ is achieved by optimizing the balance between the carboxyl group content and scaffold conductivity of GA. Importantly, the GA sensor greatly outperforms all reported carbon-based $\mathrm{H}_{2} \mathrm{O}_{2}$ sensors and is superior to enzymatic ones

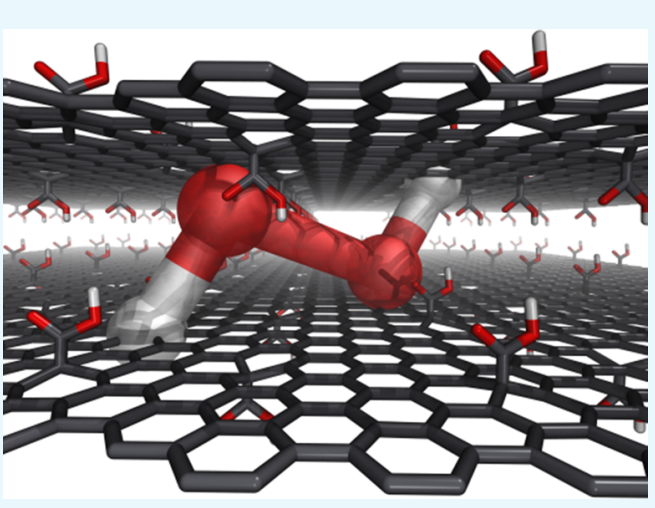
because of its simple immobilization, low cost, and uncompromised sensitivity even after continuous operation for 7 days. In addition, GA-based sensing electrodes remain highly selective in the presence of interferents such as ascorbic acid, paracetamol, and glucose, as well as complex matrices such as milk. GA-based sensors thus have considerable potential for use in practical industrial sensing technologies.

\section{INTRODUCTION}

Hydrogen peroxide $\left(\mathrm{H}_{2} \mathrm{O}_{2}\right)$ is widely used in industry; its worldwide production has been projected to reach $6 \times 10^{6}$ tons by 2024, making it among the 100 most important bulk chemicals. ${ }^{1,2}$ Sectors that use $\mathrm{H}_{2} \mathrm{O}_{2}$ extensively include the textile, pulp, paper, semiconductor, food, and cosmetic industries. However, $\mathrm{H}_{2} \mathrm{O}_{2}$ is cytotoxic, ${ }^{3}$ so its presence in commercial products is strictly monitored. Indicative concentration limits range from $12 \%$ for hair care products to $0.1 \%$ for oral hygiene commodities, and zero-tolerance in food products. ${ }^{4}$ Moreover, 3-10 wt \% solutions of $\mathrm{H}_{2} \mathrm{O}_{2}$ in water are used around the world as an antiseptic. Consequently, there is a growing demand for simple, fast, sensitive, and inexpensive sensors for $\mathrm{H}_{2} \mathrm{O}_{2}$ detection.

Conventional spectroscopic techniques such as spectrophotometry, ${ }^{5-8}$ fluorescence spectroscopy, ${ }^{9-11}$ and chemiluminescence spectroscopy ${ }^{12-16}$ are highly sensitive but require complex and expensive instrumentation and long detection times. Moreover, spectrophotometric techniques are often incompatible with real samples containing dispersed particles or dissolved species that may scatter or absorb light, affecting the measurement. In contrast, electrochemical methods could potentially enable simple, rapid, sensitive, and cost-effective $\mathrm{H}_{2} \mathrm{O}_{2}$ detection, avoiding the major drawbacks of spectroscopic methods. ${ }^{17-20}$ In principle, electrochemical $\mathrm{H}_{2} \mathrm{O}_{2}$ detection could be achieved using a conventional solid-state electrode such as the glassy carbon electrode (GCE). However, this approach is limited by high redox overpotentials and a lack of selectivity, leading to inaccurate determination of $\mathrm{H}_{2} \mathrm{O}_{2}$ in real samples. These issues are exacerbated when other electroactive species are present (e.g., ascorbate, urate, glucose, paracetamol, or bilirubin). Therefore, there is an ongoing effort to rationally design new materials for $\mathrm{H}_{2} \mathrm{O}_{2}$ detection that combine sensitivity, selectivity, and favorable kinetics with a long life cycle and cost-effectiveness.

One of the most successful approaches resulting from this effort relies on the modification of electrodes with enzymes. ${ }^{21}$ Because most active sites in enzymes are highly specific for

Received: September 5, 2019

Accepted: November 4, 2019

Published: November 15, 2019 

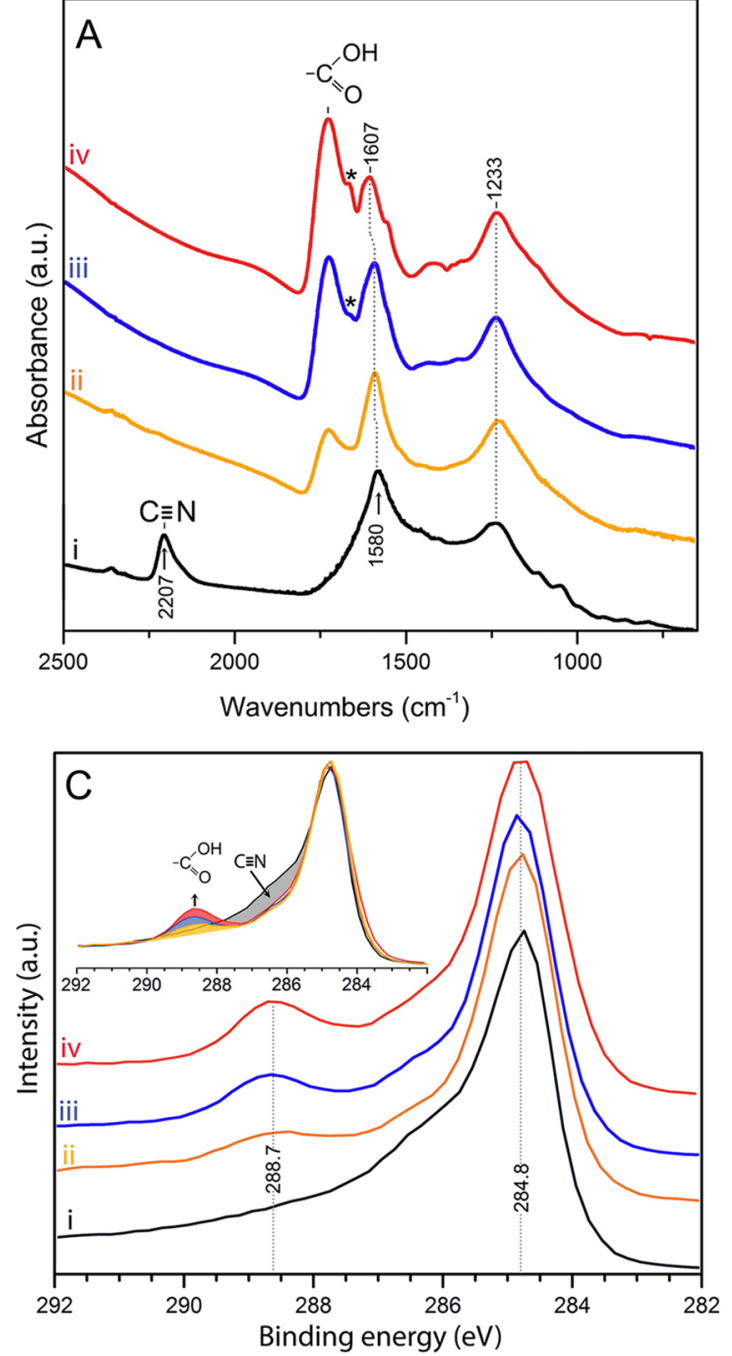

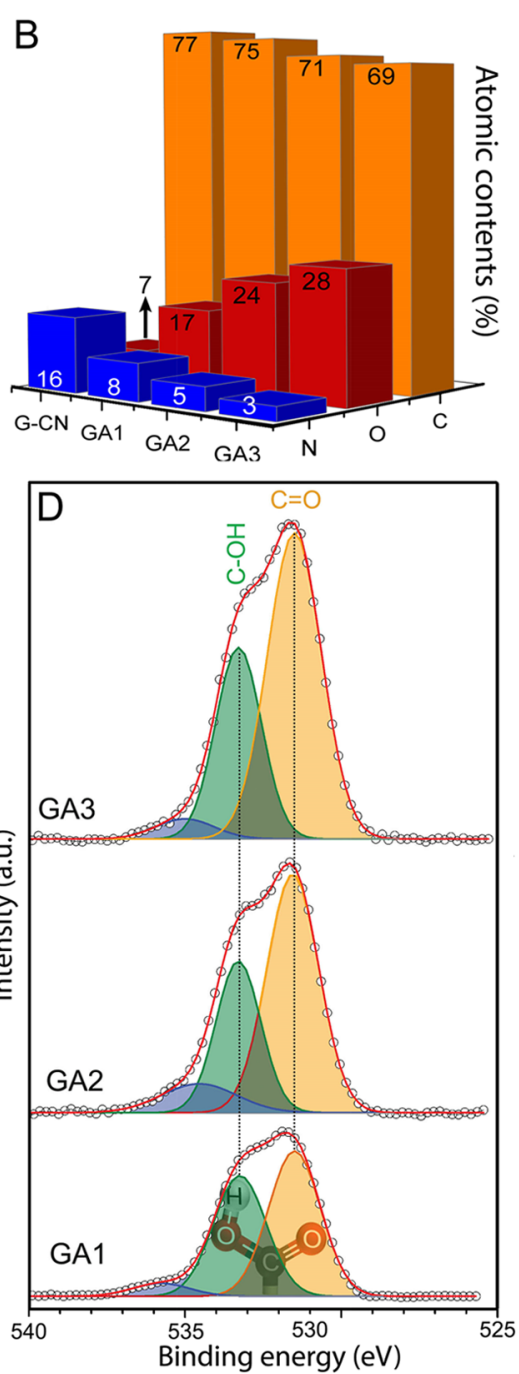

Figure 1. (A) FTIR spectra of (i) the starting cyanographene, G-CN, and the three carboxyl derivatives with increasing $-\mathrm{COOH}$ contents: (ii) GA1, (iii) GA2, and (iv) GA3. (B) Atomic concentrations of C, O, and N retrieved from XPS survey analysis for the studied samples (numbers are subjective to an error of $\pm 5 \%$ ). (C) C 1s HR-XPS spectra of (i) the starting cyanographene (G-CN), and the carboxyl derivatives: (ii) GA1, (iii) GA2, and (iv) GA3. The inset shows a superposition of the same spectra normalized based on the intensity of the main C-C component at 284.8 eV. (D) Deconvoluted O 1s HR-XPS spectra of the three GA derivatives.

their target reactions, enzymatic sensors are generally very selective. However, they have notable drawbacks including relatively high costs, instability toward variation in temperature and $\mathrm{pH}$, complicated immobilization procedures, and an inherent tendency toward denaturation of the enzyme, ${ }^{22}$ which hinders their commercial application. Because of these limitations, there is growing interest in non-enzymatic alternatives, many of which incorporate nanostructured metals and metal oxides or hybrids thereof with carbon nanomaterials. ${ }^{23}$ Carbon materials are widely used in electrochemistry because of their low cost, wide potential window, high stability, conductivity, and remarkable selectivity for diverse redox reactions, including $\mathrm{CO}_{2}$ and $\mathrm{O}_{2}$ reduction. ${ }^{24-34}$ Their potential as electrochemical sensors is enhanced by their ready modification (in contrast to metal electrodes) and high surface area, which favors analyte adsorption and accumulation. $^{24,35}$

Both carbon nanotubes $(\mathrm{CNTs})^{36-39}$ and graphene $\mathrm{e}^{1,40-43}$ have thus been used as electrodes for electrochemical $\mathrm{H}_{2} \mathrm{O}_{2}$ detection. For example, a $\mathrm{H}_{2} \mathrm{O}_{2}$ electrosensor based on chemically reduced graphene oxide $(\mathrm{CR}-\mathrm{GO})^{41}$ reportedly outperformed both $\mathrm{CNTs}^{41}$ and a bare GCE. ${ }^{40}$ Moreover, the CR-GO system was able to operate at concentrations between 0.05 and $1500 \mu \mathrm{mol}$ with a sensitivity of $82.35 \mu \mathrm{A} \mathrm{cm} \mathrm{cm}^{-2} \mathrm{mM}^{-1}$. While GO must be reduced to convert it from an insulator into a conductor, reduction changes the material's property in some undesirable ways, by increasing its hydrophobicity and removing functional groups. This increases its tendency to aggregate and limits its solvation in water and accessibility to analyte molecules. ${ }^{44,45}$ To circumvent these problems, Woo et al. ${ }^{46}$ used MWCNTs as bridges and pillars separating the graphene sheets, creating a conductive network and preventing their aggregation. However, the resulting sensor exhibited a lower sensitivity $\left(32.91 \mu \mathrm{A} \mathrm{cm}^{-2} \mathrm{mM}^{-1}\right)$ than the original CRGO system, probably because the surface hydrophobicity and the lack of chemical functionalities for binding $\mathrm{H}_{2} \mathrm{O}_{2}$ were not adequately addressed. In another attempt to boost the performance of GO-based electrosensors, Wang et al. ${ }^{47}$ covalently grafted tyrosine onto GO and simultaneously reduced it by heating at $100{ }^{\circ} \mathrm{C}$ for $24 \mathrm{~h}$ with no added conjugation agents. The resulting material had a wide linear $\mathrm{H}_{2} \mathrm{O}_{2}$ detection range (100 to $2100 \mu \mathrm{M}$ ), a detection limit of 


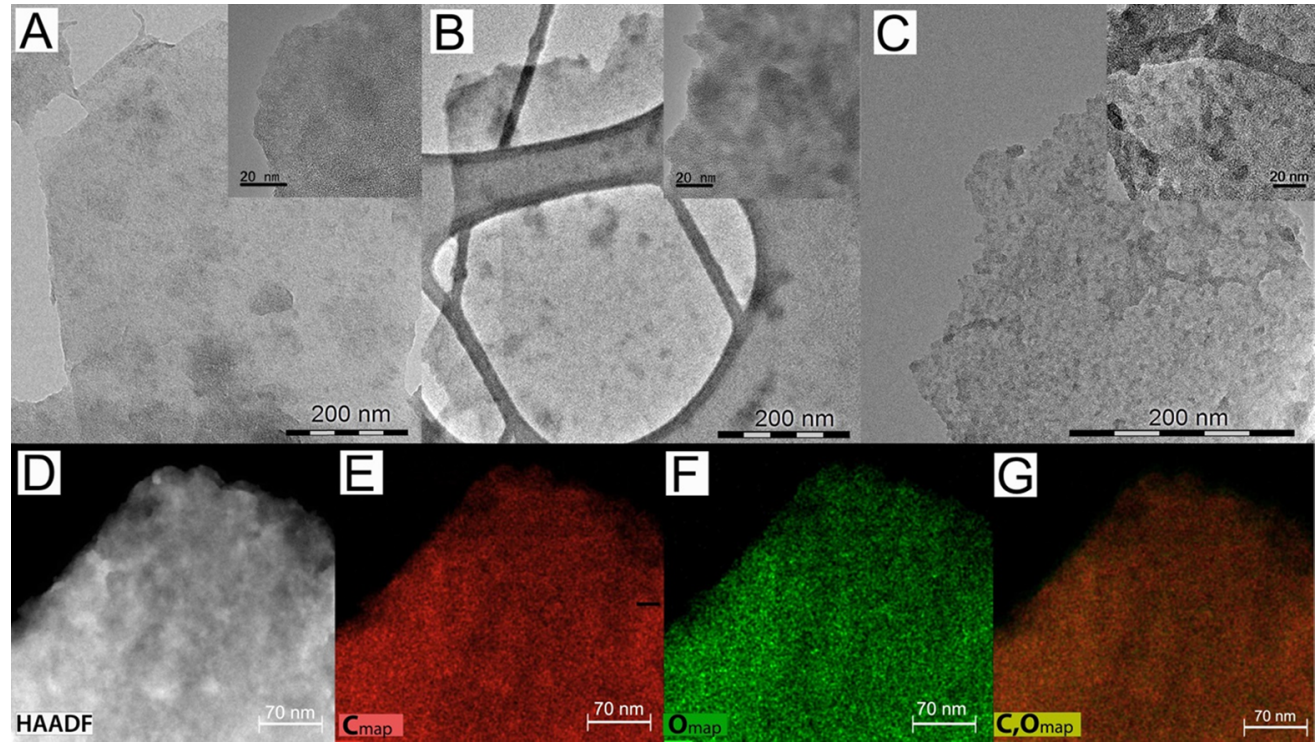

Figure 2. Transmission electron micrographs of the three graphene-acid derivatives (A) GA1, (B) GA2, and (C) GA3. The insets show higherresolution images of the samples. (D) Scanning TEM-high-angle annular dark-field (HAADF) image and energy-dispersive X-ray spectroscopy (EDS) elemental mappings for (E) carbon and (F) oxygen in GA1; (G) combined carbon-oxygen map.

$80 \mu \mathrm{M}$, and a sensitivity of $69.07 \mu \mathrm{A} \mathrm{cm} \mathrm{cm}^{-1}$, which was still lower than that of pristine CR-GO.

Heteroatom doping offers another way for improving the performance of graphene-based $\mathrm{H}_{2} \mathrm{O}_{2}$ sensors. Boron-doped graphene nanosheets were recently reported as carbon-based catalysts for $\mathrm{H}_{2} \mathrm{O}_{2}$ detection with a superior sensitivity of 266.7 $\mu \mathrm{A} \mathrm{cm}{ }^{-2} \mathrm{mM}^{-1}$. However, while their linear range is wide (1000-20,000 $\mu \mathrm{M})$, its lower bound is very high. ${ }^{48}$ The development of carbon-based catalysts for $\mathrm{H}_{2} \mathrm{O}_{2}$ sensing is probably hampered by the dense but uncontrolled functionalization of $\mathrm{GO},{ }^{49}$ which makes it an insulator, and by the low reactivity of pristine graphene. ${ }^{50,51}$ Overcoming these challenges will require an up-scalable product combining hydrophilicity with conductivity and selective functionalization for enhanced binding and reduction of $\mathrm{H}_{2} \mathrm{O}_{2}$. This could be achieved through controlled derivatization that avoids functional groups that do not contribute to sensing and thus reduce the graphene system's conductivity without providing any commensurate benefit.

Based on these considerations and the proton-catalyzed $\mathrm{H}_{2} \mathrm{O}_{2}$ reduction mechanism of peroxidases, ${ }^{52}$ we propose a selectively and densely functionalized carboxylic graphene derivative (graphene acid, ${ }^{53} \mathrm{GA}$ ) as a two-dimensional proton source and conductive electrode for binding and detecting $\mathrm{H}_{2} \mathrm{O}_{2}$. Whereas GO synthesis requires concentrated acids and strong oxidizers, GA is prepared under mild conditions that preserve the graphene sheets' conductivity and avoid introducing functionalities that are not useful for $\mathrm{H}_{2} \mathrm{O}_{2}$ sensing. These properties were exploited to develop a GAbased electrochemical $\mathrm{H}_{2} \mathrm{O}_{2}$ sensor with sensitivity as high as $525 \mu \mathrm{A} \mathrm{cm}^{-2} \mathrm{mM}^{-1}$, setting a new benchmark in the field (see Table S1 for detailed comparisons). Analyses with inductively coupled plasma mass spectrometry (ICP-MS) precluded any metal impurity-induced activity. Remarkably, the GA-based sensor's sensitivity surpassed that of enzymatic sensors ${ }^{54}$ while remaining stable even after a week of continuous operation. It was also very robust, retaining high sensitivity and selectivity for $\mathrm{H}_{2} \mathrm{O}_{2}$ in the presence of interferents such as ascorbic acid, paracetamol, and glucose, or when tested in complex matrices such as milk.

\section{RESULTS AND DISCUSSION}

GA was synthesized from fluorographene (FG), a stable, stoichiometric, and well-defined graphene derivative, which was subjected to nucleophilic substitution with $\mathrm{NaCN}$, yielding fluorine-free G-CN with $15 \%$ nitrile group coverage. ${ }^{53}$ The $-\mathrm{CN}$ groups were then quite selectively converted into $-\mathrm{COOH}$ groups by acid hydrolysis with $\mathrm{HNO}_{3}$, yielding densely functionalized GA. ${ }^{53}$ By varying the concentration of $\mathrm{HNO}_{3}$ in the acid hydrolysis step, GA samples with varying carboxyl contents (GA1, GA2, and GA3) were obtained. Pristine G-CN displayed a pronounced IR band at $2207 \mathrm{~cm}^{-1}$ (Figure 1A, spectrum i) due to the triple bond of the covalently attached $\mathrm{C} \equiv \mathrm{N}$ groups. Upon treatment with $20 \%$ $\mathrm{HNO}_{3}$, the $\mathrm{C} \equiv \mathrm{N}$ band disappeared, indicating the replacement of the nitrile groups with carboxyls (GA1, Figure 1, spectrum ii). Raising the acid concentration during the hydrolysis step to $40 \%$ (GA2) or $65 \%$ (GA3) increased the content of $-\mathrm{COOH}$ groups, as indicated by the increasing intensity of the band at $1726 \mathrm{~cm}^{-1}$ (Figure 1A, spectra ii-iv). The increasing $-\mathrm{COOH}$ content in the GA1-GA3 samples also affected the $\mathrm{C}=\mathrm{C}$ bands (aromatic ring stretching vibrations), ${ }^{55,56}$ which appeared at $1580 \mathrm{~cm}^{-1}$ in the G-CN spectrum. Specifically, as the $-\mathrm{COOH}$ content increased, the aromaticity and extent of conjugation decreased, leading to a shift of the band to $1607 \mathrm{~cm}^{-1}$. The broad band with maximum intensity centered at $1233 \mathrm{~cm}^{-1}$ is ascribed to in-plane carbon ring and carboxyl vibrations. ${ }^{57}$

Atomic compositions retrieved from XPS survey analysis showed that acid hydrolysis reduced the samples' content of nitrogen and increased that of oxygen (Figure 1B). HR-XPS data for the $\mathrm{C}$ 1s region also indicated that the increasing $\mathrm{O}$ content was attributable to carboxyl-type carbons because of the very characteristic component evolving at $288.7 \mathrm{eV}$ (Figure $1 \mathrm{C}$ and inset). ${ }^{58}$ Additionally, the $\mathrm{C} \equiv \mathrm{N}$ component (highlighted in gray in the inset of Figure $1 \mathrm{C}$ ) of G-CN was absent in the spectra of GA1, GA2, and GA3, all of which had near- 

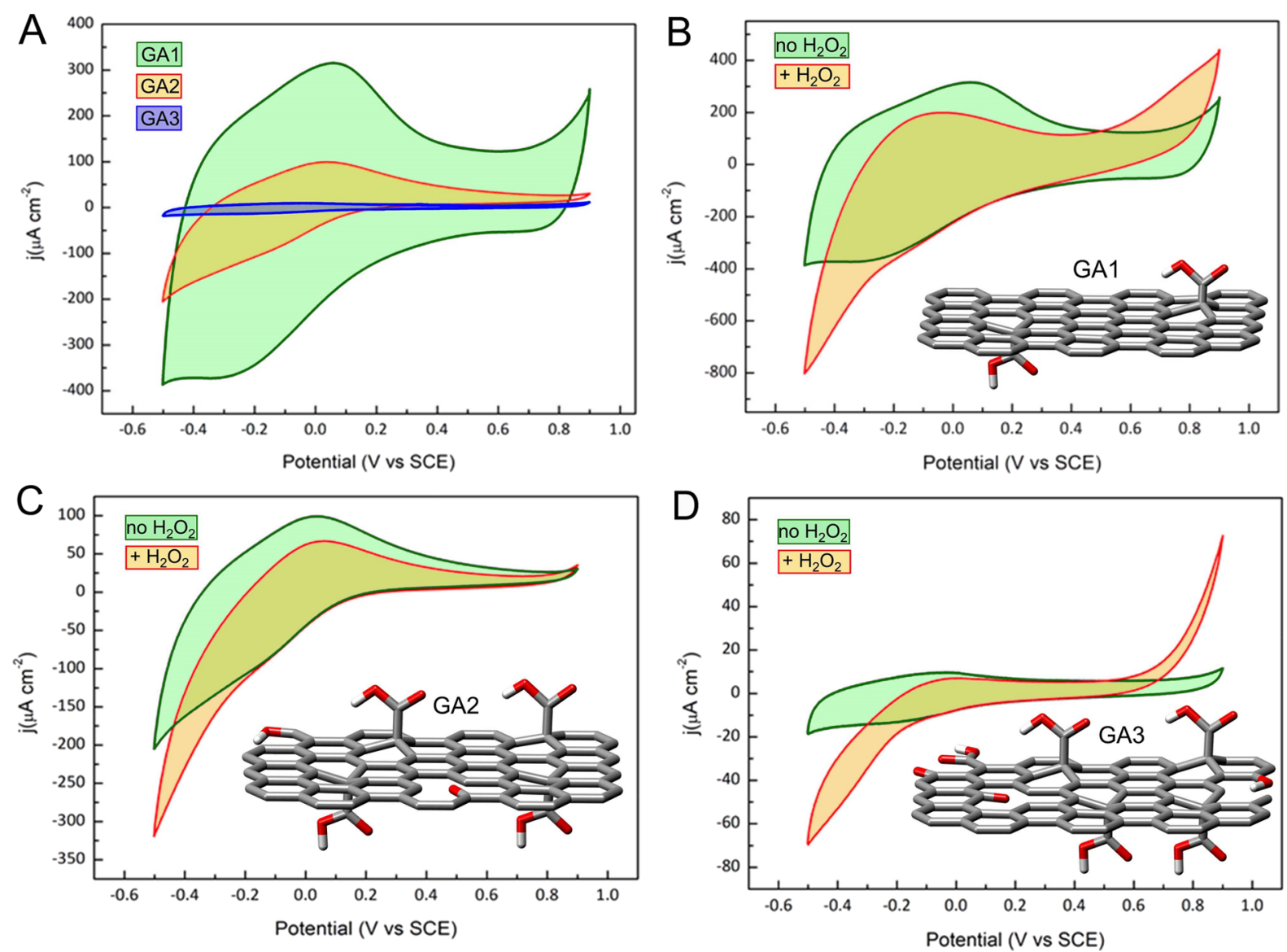

Figure 3. (A) Cyclic voltammetry (CV) cathodic scans of glassy carbon electrodes (GCEs) modified with GA1, GA2, and GA3. Cyclic voltammetry cathodic scans of GCEs modified with (B) GA1, (C) GA2, and (D) GA3 in the absence and in the presence of $5 \mathrm{mM} \mathrm{H}_{2} \mathrm{O}_{2}$. The insets in panels (B) $-(\mathrm{D})$ show tentative structures of the GA1-GA3 products. All CVs were recorded in $0.10 \mathrm{M}$ PBS buffer solution at pH 7.0 under $\mathrm{Ar}$ at a scan rate of $0.05 \mathrm{~V} \mathrm{~s}^{-1}$.

identical $\mathrm{C} \equiv \mathrm{N}$ regions. This corroborated the IR data, suggesting that almost all nitrile groups hydrolyzed, even in GA1. The $\mathrm{O}$ and $\mathrm{N}$ contents in the G-CN and GA samples are probably due to the side reactions during the synthesis of G$\mathrm{CN}$ from $\mathrm{FG}$, which is performed in $\mathrm{DMF}$ at $130{ }^{\circ} \mathrm{C}$; at this temperature, DMF decomposes into amines that are reactive toward FG. ${ }^{53}$ The $\mathrm{O}$ 1s region of the deconvoluted HR-XPS spectrum of the GA1 sample (Figure 1D) contains two main components (the third minor one is ascribed to chemisorbed $\mathrm{O}_{2}$ and $\mathrm{H}_{2} \mathrm{O}$ ). The main components in GAl are almost equal in area, which is in agreement with the presence of $\mathrm{O}=\mathrm{C}-$ $\mathrm{OH}$ (carboxyl) groups. ${ }^{59}$ While acidic hydrolysis of the nitrile groups with $\mathrm{HNO}_{3}$ led to a relatively selective carboxylation in sample GA1, the higher $\mathrm{HNO}_{3}$ concentrations used to prepare GA2 and GA3 induced less selective oxidation of the graphene skeleton, as indicated by the increasing $-\mathrm{C}=\mathrm{O}$ over the $-\mathrm{C}-$ $\mathrm{OH}$ component (Figure 1D) and the additional $\mathrm{C}=\mathrm{O}$ IR bands indicated by asterisks in Figure 1A.

The quality of the sheets in samples GA1-GA3 was probed by HR-TEM (Figure $2 \mathrm{~A}-\mathrm{C}$ and insets). The use of nitric acid concentrations above that required for selective nitrile hydrolysis apparently reduced the graphene skeleton's integrity: GA2 and GA3 had more small fragments of material and defects than GA1 (Figure 2B,C and insets).The HAADFSTEM image and elemental mapping of carbon and oxygen in the GA1 sample (Figure 2D-G) clearly show the homogeneous and dense atomic distribution of oxygen on its sheets.
The higher quality flake structure of the GA1 sample was further probed with scanning electron microscopy (Figure S1).

The electrocatalytic properties of the GA derivatives were evaluated by cyclic voltammetry (CV) in Ar-saturated PBS buffer solution at $\mathrm{pH} 7.0$ because $\mathrm{H}_{2} \mathrm{O}_{2}$ is less readily detected at neutral $\mathrm{pH}$ than under basic or acidic conditions, making for a more stringent and practically relevant test of performance. ${ }^{60}$ In all the three investigated cases, an anodic/cathodic couple of broad peaks was detected that was attributed to the redox processes of the carboxyl groups, according to the results described in Figure S3. A significant decline in current density was observed upon moving from GA1 to GA3 (Figure 3A), in agreement with the previous data, regarding the lower aromatic character and sheet quality of GA2 and GA3, since both factors would tend to reduce conductivity. All GA samples exhibited a stronger cathodic current density in the presence of the $\mathrm{H}_{2} \mathrm{O}_{2}$ analyte (Figure $3 \mathrm{~B}-\mathrm{D}$ ), with an onset potential of $-0.15 \mathrm{~V}$ after the addition of $5 \mathrm{mM} \mathrm{H} \mathrm{H}_{2}$. This suggested that all GAs were active in $\mathrm{H}_{2} \mathrm{O}_{2}$ reduction and thus detection. Nevertheless, the $\mathrm{CV}$ scans showed that the current density response at $-0.4 \mathrm{~V}$ for GA1 $\left(-627 \mu \mathrm{A} \mathrm{cm}^{-2}\right)$ was much stronger than that for GA2 $\left.(-231 \mu \mathrm{A} \mathrm{cm})^{-2}\right)$ or GA3 $\left(-53 \mu \mathrm{A} \mathrm{cm}^{-2}\right)$. This confirmed the importance of mild acid treatment (i.e., selective nitrile group hydrolysis). Interestingly, the current density response upon $\mathrm{H}_{2} \mathrm{O}_{2}$ addition using $\mathrm{GO}$ was marginal, as shown by the very small increase of current density in the CVs upon addition of $\mathrm{H}_{2} \mathrm{O}_{2}$ (Figure $\mathrm{S} 2 \mathrm{~A}$ ). 

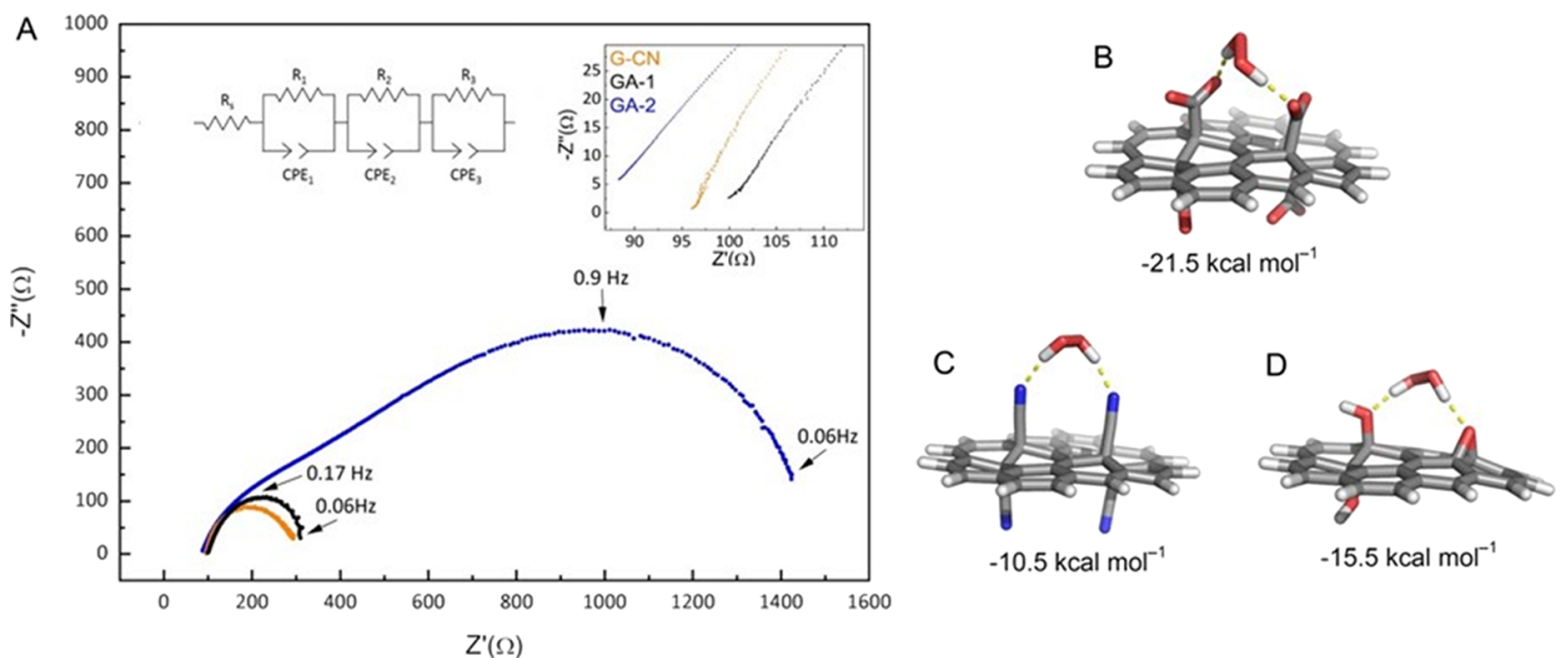

Figure 4. (A) Nyquist plots obtained for G-CN (orange line), GA1 (black line), and GA2 (blue line) using a rotating disk electrode in an Arsaturated PBS $0.1 \mathrm{M}(\mathrm{pH}=7)$ electrolyte with $25 \mathrm{mmol}$ of $\mathrm{H}_{2} \mathrm{O}_{2}$ at $-0.4 \mathrm{~V}$ vs SCE; the left inset shows the equivalent circuit used for fitting where $R_{\mathrm{s}}$ is the solution resistance, and the subsequent (resistor, constant phase element, CPE) components are associated with the charge-transfer resistance in parallel with the double layer capacitance $\left(R_{1}, \mathrm{CPE} 1\right)$ and the resistance related to diffusion processes $\left(R_{2}, \mathrm{CPE} 2\right.$ and $\left.R_{3}, \mathrm{CPE} 3\right)$. The right inset shows a magnification of the high frequency part of the Nyquist plot where the charge-transfer processes take place. (B-D) Optimized structures of complexes of graphene derivatives (in water) (B, GA; C, G-CN; and D, GO) with $\mathrm{H}_{2} \mathrm{O}_{2}$. The hydrogen bonds between $\mathrm{H}_{2} \mathrm{O}_{2}$ and graphene functional groups are indicated by yellow dashed lines. The interaction energies in kcal mol ${ }^{-1}$ between $\mathrm{H}_{2} \mathrm{O}_{2}$ and graphene derivatives are stated below each structure.
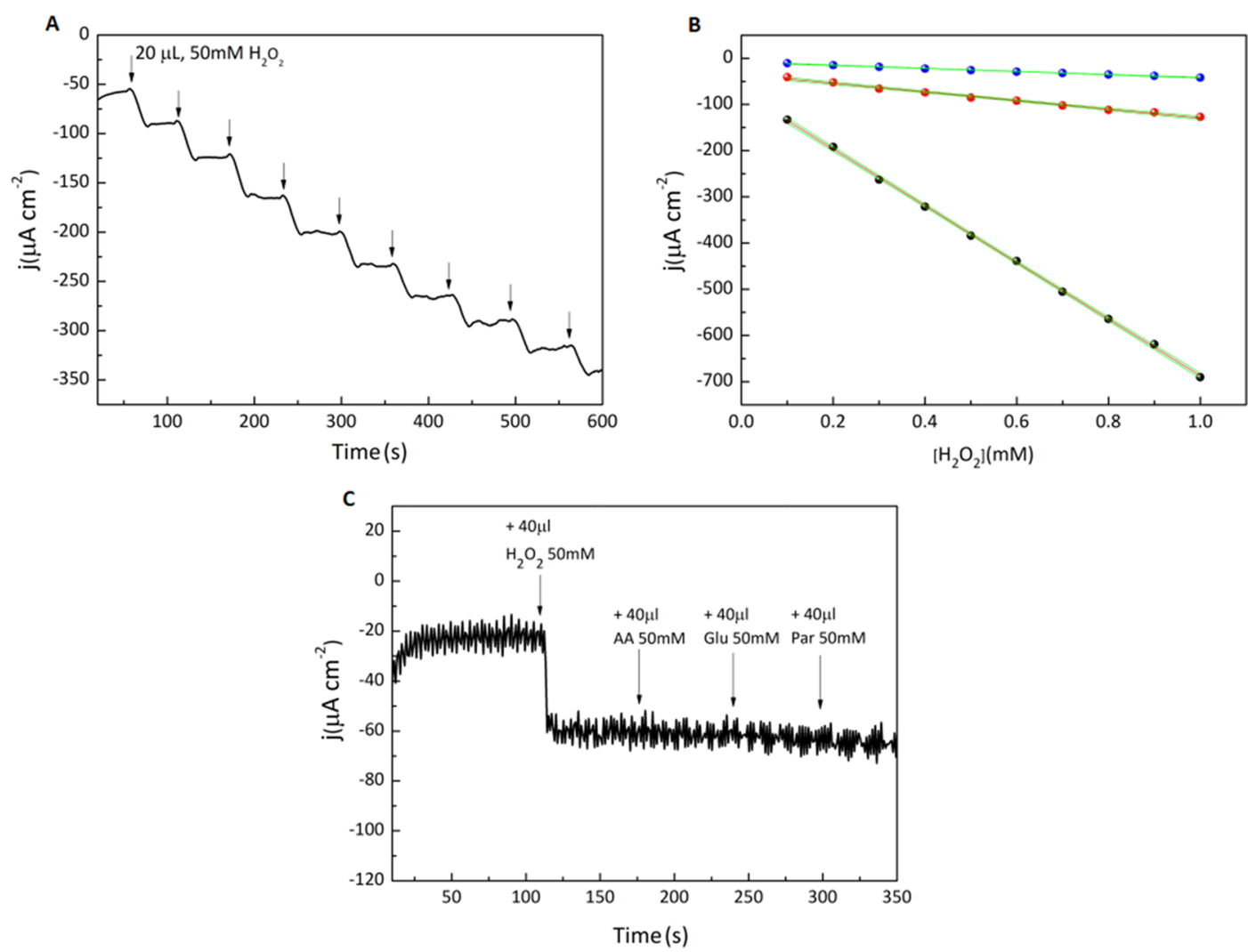

Figure 5. (A) Chronoamperometry plot for repeated additions of $20 \mu \mathrm{L}$ of a $50 \mathrm{mM} \mathrm{H}_{2} \mathrm{O}_{2}$ solution to $10 \mathrm{~mL}$ of $0.1 \mathrm{M}$ PBS solution using the GA1coated GCE. Arrows indicate additions of $\mathrm{H}_{2} \mathrm{O}_{2}$. (B) Calibration plots obtained from the amperometric responses presented in panel (A) for GA1 (black dots), GA2 (red dots), and GA3 (blue dots). The red line is the linear fit to the data and the green lines indicate the $95 \%$ confidence bands. (C) Chronoamperometry of GAl at $-0.4 \mathrm{~V}$ showing sequential $40 \mu \mathrm{L}$ additions of $50 \mathrm{mM}$ solutions of $\mathrm{H}_{2} \mathrm{O}_{2}$, ascorbic acid (AA), glucose (Glu), and paracetamol (Par).

To better understand the electrochemical properties of GA electrodes, we performed EIS measurements using an optimized Nafion-based ink containing the GA samples. The instability of the GA3 ink caused the detachment from the electrode and the leaching in the aqueous solution, preventing its characterization. This was probably due to the very high 
hydrophilicity of GA3, which resulted from its high content of oxygen groups and defects, as well as its comparatively small flake size (Figure 2C). Based on the Nyquist plots for GA1 and GA2 (Figure 4A) and the corresponding equivalent circuit (EC) used to fit the EIS data, the GA1-modified electrode exhibited a charge-transfer resistance of $8 \Omega \mathrm{cm}^{2}$, three times lower than that of GA2 (Table S2), confirming the importance of conductivity for imparting high performance. In control experiments, the non-carboxylated GA precursor (G-CN) exhibited a worse $\mathrm{H}_{2} \mathrm{O}_{2}$ sensing performance with respect to GA1 (Figure S3) despite its higher conductivity, as previously reported. ${ }^{61}$ The latter is also evidenced here from the lower charge-transfer resistance $\left(R_{1}=5 \Omega \mathrm{cm}^{2}\right.$, as simulated from the EIS data in Figure 4A and Table S2) than that of GA1. G-CN also demonstrated lower stability of the response (Figure S4) and a narrower linear range, being unable to operate at concentrations below $0.1 \mathrm{mM} \mathrm{H}_{2} \mathrm{O}_{2}$. The electrochemical response of the GA-CN product treated with a lower amount of $\mathrm{HNO}_{3}$ than the GAl and thus being a mixed-functionalized graphene carrying both $-\mathrm{CN}$ and $-\mathrm{COOH}$ groups (see IR spectra in Figure S3 inset) was also inferior to GA's response (Figure S3). These results demonstrated the key role of $-\mathrm{COOH}$ groups tethered onto a sufficiently conductive substrate. Indeed, catalytic $\mathrm{H}_{2} \mathrm{O}_{2}$ reduction by enzymes (i.e., peroxidases) is triggered by the supply of protons from amino acid residues in the enzyme's $\mathrm{H}_{2} \mathrm{O}_{2}$ binding pocket, together with electron injection from sacrificial donors. ${ }^{52}$

Furthermore, DFT calculations (Figure 4B-D) indicated that $\mathrm{H}_{2} \mathrm{O}_{2}$ binds more tightly to $\mathrm{GA}\left(-21.5 \mathrm{kcal} \mathrm{mol}^{-1}\right)$ than to $\operatorname{GCN}\left(-10.2 \mathrm{kcal} \mathrm{mol}^{-1}\right)$ or GO $\left(-15.5 \mathrm{kcal} \mathrm{mol}^{-1}\right)$ because strong hydrogen bonds are formed between the $\mathrm{OH}$ groups of $\mathrm{H}_{2} \mathrm{O}_{2}$ and the carboxylate groups of GA. It should be highlighted that ICP-MS analyses confirmed that metal impurities in the GA1 product are not responsible for any catalytic activity (see Table S3 for results and discussion).

To further evaluate the sensing performance of GA1, we conducted chronoamperometry measurements at $-0.4 \mathrm{~V}$ versus SCE by sequentially adding $20 \mu \mathrm{L}$ portions of a 50 $\mathrm{mM} \mathrm{H} \mathrm{H}_{2} \mathrm{O}_{2}$ solution to a $10 \mathrm{~mL} 0.1 \mathrm{M}$ PBS solution (Figure $5 \mathrm{~A})$. The value $-0.4 \mathrm{~V}$ versus SCE was chosen as the working potential as it produced the best response from the sensor in terms of sensitivity and the linear range. Calibration plots for the three samples derived from chronoamperometry data revealed a linear relationship between the current density and the $\mathrm{H}_{2} \mathrm{O}_{2}$ concentration over the investigated range (Figure $5 \mathrm{~B})$. The obtained values reflect the previously observed trend, with GA1 displaying the highest average sensitivity $(525 \pm 105$ $\mu \mathrm{A} \mathrm{cm} \mathrm{cm}^{-2} \mathrm{mM}^{-1}$, while GA2 and GA3 showed progressively worse performance, with average sensitivities of $94 \pm 25$ and $40 \pm 9 \mu \mathrm{A} \mathrm{cm}^{-2} \mathrm{mM}^{-1}$, respectively. The high sensitivity of GA1, exceeding that of GA3 by more than one order of magnitude, correlated with its excellent electron transfer properties, which resulted from the optimized synthetic protocol. By repeating the experiment with different concentration windows, we estimated the sensor's linear range; $\mathrm{H}_{2} \mathrm{O}_{2}$ could be detected with the quoted sensitivities at concentrations between 20 and $2000 \mu \mathrm{M}$, making GA-based sensors potentially suitable for several biological and industrial applications. Remarkably, the sensitivity of GA1 substantially exceeded that of other recently reported graphene-based sensors: ${ }^{43,46,47}$ it was twice that of the previous record holder (B-doped graphene, ${ }^{48}$ with a sensitivity of $266 \mu \mathrm{A} \mathrm{cm} \mathrm{cm}^{-2}$ $\mathrm{mM}^{-1}$ ) and even higher than some enzymatic systems. ${ }^{54} \mathrm{~A}$ detailed comparison of the performance of state-of-the-art carbon-based sensors is available in Table S1.

GA1 was tested further under conditions more closely resembling real applications to validate its reproducibility/ stability, selectivity, and robustness when exposed to real, complex matrices. Its reproducibility and stability in the presence of possible $\mathrm{H}_{2} \mathrm{O}_{2}$ degradation products such as oxygen radicals were evaluated by performing amperometric tests at $-0.4 \mathrm{~V}$ for 7 consecutive days during which its average sensitivity remained unchanged.

Another crucial determinant of a $\mathrm{H}_{2} \mathrm{O}_{2}$ sensor's reliability is its response to interference by oxidizable compounds commonly present in commercial samples such as ascorbic acid, glucose, and paracetamol. Figure 5C shows the amperometric response of $\mathrm{GAl}$ to $\mathrm{H}_{2} \mathrm{O}_{2}$ in the presence of these three compounds. The current density increased steeply after the addition of $\mathrm{H}_{2} \mathrm{O}_{2}$, but no such increase followed the addition of the interfering substances, demonstrating the high selectivity of GA1 for $\mathrm{H}_{2} \mathrm{O}_{2}$. The general robustness and reliability of the GA1 sensor was then evaluated in a realistic test case by adding commercial milk to the electrolyte solution while performing chronoamperometry tests. Milk was chosen because $\mathrm{H}_{2} \mathrm{O}_{2}$ is widely used in dairy production to inhibit microbial proliferation and milk spoilage and its concentration must be strictly monitored for safety reasons. Milk is also a complex mixture of different compounds, and it is a particularly demanding system for benchmarking $\mathrm{H}_{2} \mathrm{O}_{2}$ sensors. Strikingly, the current density response remained utterly unaffected by the addition of milk (Figure S5). These results clearly showed that $\mathrm{GA}$ is a promising candidate for sensitive, selective, cheap, and reliable $\mathrm{H}_{2} \mathrm{O}_{2}$ sensors suitable for the testing of commercial products bearing complex matrices.

\section{CONCLUSIONS}

We demonstrated that graphene acid, a selectively carboxylated and conductive graphene derivative, acts as a carbon-based catalyst for the electroreduction and detection of $\mathrm{H}_{2} \mathrm{O}_{2}$ whose sensitivity $\left(525 \mu \mathrm{A} \mathrm{cm}^{-2} \mathrm{mM}^{-1}\right)$ markedly exceeds that of all previously reported carbon-based sensors and competes even that of enzymatic ones. However, unlike the delicate enzymatic sensors, GA's sensitivity remained uncompromised over a week of a continuous operation. This unprecedented performance is attributable to its stable carbon-based all-covalent nature, the high affinity of carboxyl groups for $\mathrm{H}_{2} \mathrm{O}_{2}$ (as revealed by DFT calculations), and their abundance in the material, which enables the efficient proton-assisted $\mathrm{H}_{2} \mathrm{O}_{2}$ electroreduction. When tested in realistic scenarios featuring interfering species and complex matrices such as milk, the graphene-acid electrosensor exhibited high selectivity and retained its sensitivity. Given the material's proven biocompatibility ${ }^{53}$ and the availability of a high-yielding and scalable synthetic protocol, these results may pave the way to the development of a previously unexplored class of carbon-based non-enzymatic commercial sensors for $\mathrm{H}_{2} \mathrm{O}_{2}$ detection.

\section{EXPERIMENTAL SECTION}

Reagents, Materials, and Methods. Nafion 117 solution (5 wt \%), $\mathrm{NaCN}$ (p.a. $\geq 97 \%$ ), and graphite fluoride (>61 wt \% $\mathrm{F}, \mathrm{C}_{1} \mathrm{~F}_{1.1}$ ) were purchased from Sigma-Aldrich. Acetone (pure) and ethanol (absolute) were purchased from Penta. Amine-free dimethylformamide (DMF) and nitric acid (Analpure, 65\%) 
were obtained from Lach-Ner. All aqueous solutions were prepared with ultrapure water $\left(18 \mathrm{M} \Omega \mathrm{cm}^{-1}\right)$.

Synthesis of Cyanographene (Precursor of GA). Cyanographene (G-CN) was synthesized according to previously reported procedures, with small modifications (see Supporting Information). ${ }^{53}$

Synthesis of Graphene Acid Samples. Nitric acid was added to aqueous suspensions of G-CN to achieve final $\mathrm{HNO}_{3}$ concentrations of 5, 20, 40, or 65 wt \% (for GA-CN, GA1, GA2, and GA3, respectively), and the resulting suspensions were heated at $100{ }^{\circ} \mathrm{C}$ with stirring $(500 \mathrm{rpm})$ for $24 \mathrm{~h}$. For the case of GA-CN, reflux was applied for $30 \mathrm{~min}$. The product was isolated and washed with $\mathrm{H}_{2} \mathrm{O}$ by centrifugation. When it stopped precipitating after a few washing steps, acidic water $(\mathrm{pH}=4)$ was used to protonate the material and reduce its dispersibility, inducing precipitation. Finally, dialysis was applied to obtain stable aqueous suspensions of GA1, GA2, and GA3 with a final $\mathrm{pH}$ of $\sim 3.2$ and a suspension conductivity of $\sim 150 \mu \mathrm{S} \mathrm{cm}{ }^{-1}$. It is noted that GA1 was synthesized according to ref 53 .

Electrochemical Techniques. Electrochemical tests were performed at room temperature using an Autolab $302 \mathrm{~N}$ electrochemical workstation (Metrohm). The cell setup was a three-electrode system with a $\mathrm{Pt}$ wire as the counter electrode, a saturated calomel electrode (SCE) that was separated from the solution by a bridge equipped with a Vycor frit as the reference electrode, and a GCE modified with the prepared electrocatalysts as the working electrode. The GCE was polished with 1.0 and $0.3 \mu \mathrm{m}$ alumina powders (Buehler MicroPolish) and rinsed thoroughly with deionized water. The electrode was then dried at $60{ }^{\circ} \mathrm{C}$. The ink $(10 \mu \mathrm{L})$ prepared from the different materials was then drop-cast on a clean GCE and dried in an oven at $60{ }^{\circ} \mathrm{C}$ for $30 \mathrm{~min}$. The inks were prepared at a concentration of $2.5 \mathrm{mg} \mathrm{mL}^{-1}$ using Nafion $1 \mathrm{wt}$ $\%$ in a 50:50 ethanol/water solution. The average sensitivity for each sample was determined by repeating the experiment multiple times. For electrochemical impedance spectroscopy (EIS) measurements, see the Supporting Information.

Density Functional Theory (DFT) Calculations. To model the interaction of $\mathrm{H}_{2} \mathrm{O}_{2}$ with graphene derivatives, we used finite size models based on functionalized ovalene $\left(\mathrm{C}_{32} \mathrm{H}_{14}\right)$ with a degree of functionalization of $12.5 \%$. Consequently, the GA, GCN, and GO models contained four carboxylate groups, four nitrile groups, and one epoxy and two hydroxyl groups, respectively (cf. Figure 4B-D). All geometries were optimized using the $\omega \mathrm{B} 97 \mathrm{xd}^{62}$ long-range corrected hybrid density functional with dispersion correction and Dunning's correlation-consistent polarized double- $\zeta$ basis sets (cc-pVDZ). ${ }^{63}$ The surrounding water was modeled using the polarizable solvation model (SMD). ${ }^{64}$ We considered several starting geometries of the $\mathrm{H}_{2} \mathrm{O}_{2} \cdots \mathrm{GA}$ complexes and selected the most stable for further analysis. The interaction energies $\left(E_{\text {int }}\right)$ between $\mathrm{H}_{2} \mathrm{O}_{2}$ and graphene derivatives were calculated as $E_{\text {int }}=E($ complex $)-E\left(\mathrm{H}_{2} \mathrm{O}_{2}\right)-E($ graphene derivative).

\section{ASSOCIATED CONTENT}

\section{S Supporting Information}

The Supporting Information is available free of charge on the ACS Publications website at DOI: 10.1021/acsomega.9b02881.
Synthesis of cyanographene, EIS measurements, characterization methods, supplementary tables and figures (PDF)

\section{AUTHOR INFORMATION}

\section{Corresponding Authors}

*E-mail: radek.zboril@upol.cz (R.Z.).

*E-mail: pfornasiero@units.it (P.F.).

ORCID $\odot$

Michele Melchionna: 0000-0001-9813-9753

Alberto Naldoni: 0000-0001-5932-2125

Michal Otyepka: 0000-0002-1066-5677

Radek Zbořil: 0000-0002-3147-2196

Paolo Fornasiero: 0000-0003-1082-9157

\section{Author Contributions}

The manuscript was written through contributions of all authors. All authors have given approval to the final version of the manuscript.

Notes

The authors declare no competing financial interest.

\section{ACKNOWLEDGMENTS}

The authors gratefully acknowledge financial support from the University of Trieste (program FRA 2018 - project INSIDE), the Italian Ministry for University and Research (MIUR program FFARB 2017), the INSTM consortium, the Ministry of Education, Youth and Sports of the Czech Republic (via projects CZ.1.05/2.1.00/19.0377, and CZ.02.1.01/0.0/0.0/ 16 019/0000754), and the Czech Science Foundation (project GA CR - EXPRO, 19-27454X). MO also acknowledges support by the ERC (Consolidator grant 683024 from the European Union's Horizon 2020 research and innovation program). Martin Petr (XPS), Ondřej Tomanec (HR-TEM), Cecilia Maria Reyes Perez (SEM), Radka Pechancova (ICP) and Dr. David Milde (ICP) are acknowledged for the measurements.

\section{ABBREVIATIONS}

GA, graphene acid; GCE, glassy carbon electrode; ICP-MS, inductively coupled plasma mass spectrometry; CNTs, carbon nanotubes; G-CN, cyanographene; EIS, electrochemical impedance spectroscopy; SCE, saturated calomel electrode; DFT, Density functional theory; FG, fluorographene; HR-XPS, high resolution X-ray photoelectron spectroscopy; EDS, energy-dispersive X-ray spectroscopy; HR-TEM, high resolution transmission electron microscopy; HAADF-STEM, high angle annular dark field scanning transmission electron microscopy; CV, cyclic voltammetry; IR, infra-red

\section{REFERENCES}

(1) Chen, W.; Cai, S.; Ren, Q.-Q.; Wen, W.; Zhao, Y.-D. Recent advances in electrochemical sensing for hydrogen peroxide: a review. Analyst 2012, 137, 49.

(2) Myers, R. L. The 100 most important chemical compounds: A reference guide; firsted. Greenwood Press: Westport, CT, 2007.

(3) Gülden, M.; Jess, A.; Kammann, J.; Maser, E.; Seibert, H. Cytotoxic potency of $\mathrm{H}_{2} \mathrm{O}_{2}$ in cell cultures: impact of cell concentration and exposure time. Free Radical Biol. Med. 2010, 49, $1298-1305$.

(4) Hydrogen Peroxide ICosmetics Info, http://www.cosmeticsinfo. org/ingredient/hydrogen-peroxide-0, accessed: 7 May 2019. 
(5) Nogueira, R. F. P.; Oliveira, M. C.; Paterlini, W. C. Simple and fast spectrophotometric determination of $\mathrm{H}_{2} \mathrm{O}_{2}$ in photo-Fenton reactions using metavanadate. Talanta 2005, 66, 86-91.

(6) Yang, Q.; Lu, S.; Shen, B.; Bao, S.; Liu, Y. An iron hydroxyl phosphate microoctahedron catalyst as an efficient peroxidase mimic for sensitive and colorimetric quantification of $\mathrm{H}_{2} \mathrm{O}_{2}$ and glucose. New J. Chem. 2018, 42, 6803.

(7) Peng, H.; Lin, D.; Liu, P.; Wu, Y.; Li, S.; Lei, Y.; Chen, W.; Chen, Y.; Lin, X.; Xia, X.; Liu, A. Highly sensitive and rapid colorimetric sensing platform based on water-soluble WOx quantum dots with intrinsic peroxidase-like activity. Anal. Chim. Acta 2017, 992, 128.

(8) Zhang, D.; Mao, X.; Zhang, Z.; Zhang, S.; Chen, J.; Shan, D.; Lu, $\mathrm{X}$. Fabricated nanoplatform of $\mathrm{Cu}$ (II)-functionalized mimeticperoxidase with catalytic property toward sensitive monitoring of hydrogen peroxide. Sens. Actuators, B 2019, 284, 684.

(9) Gomes, A.; Fernandes, E.; Lima, J. L. Fluorescence probes used for detection of reactive oxygen species. J. Biochem. Biophys. Methods 2005, 65, 45.

(10) Lee, J. H.; Tang, I. N.; Weinstein-Lloyd, J. B. A non-enzymatic method for the determination of hydrogen peroxide in atmospheric samples. Anal. Chem. 1990, 62, 2381.

(11) Lu, H.; Yu, C.; Zhang, Y.; Xu, S. Efficient core shell structured dual response ratiometric fluorescence probe for determination of $\mathrm{H}_{2} \mathrm{O}_{2}$ and glucose via etching of silver nanoprisms. Anal. Chim. Acta 2019, 1048, 178.

(12) Hanaoka, S.; Lin, J.-M.; Yamada, M. Chemiluminescent flow sensor for $\mathrm{H}_{2} \mathrm{O}_{2}$ based on the decomposition of $\mathrm{H}_{2} \mathrm{O}_{2}$ catalyzed by cobalt(II)-ethanolamine complex immobilized on resin. Anal. Chim. Acta 2001, 426, 57.

(13) Tang, X. Q.; Zhang, Y. D.; Jiang, Z. W.; Wang, D. M.; Huang, C. Z.; Li, Y. F. $\mathrm{Fe}_{3} \mathrm{O}_{4}$ and metal-organic framework MIL-101(Fe) composites catalyze luminol chemiluminescence for sensitively sensing hydrogen peroxide and glucose. Talanta 2018, 179, 43.

(14) Chen, H.; Lin, L.; Li, H.; Li, J.; Lin, J.-M. Aggregation-induced structure transition of protein-stabilized zinc/copper nanoclusters for amplified chemiluminescence. ACS Nano 2015, 9, 2173.

(15) Pan, F.; Wei, P.; Zhang, M.; Lu, C. Micelle modified-carbon nanosphere enhanced chemiluminescence from reactive oxygen species for the detection of hydrogen peroxide. Anal. Methods 2015, $7,5667$.

(16) Li, Y.; You, X.; Shi, X. Enhanced chemiluminescence determination of hydrogen peroxide in milk sample using metalorganic framework $\mathrm{Fe}-\mathrm{MIL}-88 \mathrm{NH}_{2}$ as peroxidase mimetic. Food Anal. Methods 2017, 10, 626.

(17) Wang, J. Electrochemical biosensors: towards point-of-care cancer diagnostics. Biosens. Bioelectron. 2006, 21, 1887.

(18) Amarnath, C. A.; Sawant, S. N. Tailoring synthesis strategies for polyaniline-prussian blue composite in view of energy storage and $\mathrm{H}_{2} \mathrm{O}_{2}$ sensing application. Electrochim. Acta 2019, 295, 294.

(19) Ebrahimi, A.; Zhang, K.; Dong, C.; Subramanian, S.; Butler, D.; Bolotsky, A.; Goodnight, L.; Cheng, Y.; Robinson, J. A. FeS -graphene $^{-}$ heterostructures: Nanofabrication-compatible catalysts for ultrasensitive electrochemical detection of hydrogen peroxide. Sens. Actuators, B 2019, 285, 631 .

(20) Liu, T.; Guo, Y.; Zhang, Z.; Miao, Z.; Zhang, X.; Su, Z. Fabrication of hollow $\mathrm{CuO} / \mathrm{PANI}$ hybrid nanofibers for nonenzymatic electrochemical detection of $\mathrm{H}_{2} \mathrm{O}_{2}$ and glucose. Sens. Actuators, B 2019, 286, 370.

(21) Mao, L.; Osborne, P. G.; Yamamoto, K.; Kato, T. Continuous On-Line Measurement of Cerebral Hydrogen Peroxide Using Enzyme-Modified Ring-Disk Plastic Carbon Film Electrode. Anal. Chem. 2002, 74, 3684.

(22) Breslow, R. Biomimetic chemistry and artificial enzymes: catalysis by design. Acc. Chem. Res. 1995, 28, 146.

(23) Bracamonte, M. V.; Melchionna, M.; Giuliani, A.; Nasi, L.; Tavagnacco, C.; Prato, M.; Fornasiero, $\mathrm{P}$. $\mathrm{H}_{2} \mathrm{O}_{2}$ sensing enhancement by mutual integration of single walled carbon nanohorns with metal oxide catalysts: the $\mathrm{CeO}_{2}$ case. Sens. Actuators, B 2017, 239, 923.
(24) McCreery, R. L. Advanced carbon electrode materials for molecular electrochemistry. Chem. Rev. 2008, 108, 2646.

(25) Duan, X.; Xu, J.; Wei, Z.; Ma, J.; Guo, S.; Wang, S.; Liu, H.; Dou, S. Metal-free carbon materials for $\mathrm{CO}_{2}$ electrochemical reduction. Adv. Mater. 2017, 29, 1701784.

(26) Gao, Z.; Zhang, Y.; Song, N.; Li, X. Biomass-derived renewable carbon materials for electrochemical energy storage. Mater. Res. Lett. 2017, 5, 69 .

(27) Rachiy, B. I.; Budzulyak, I. M.; Vashchynsky, V. M.; Ivanichok, N. Y.; Nykoliuk, M. O. Electrochemical properties of nanoporous carbon material in aqueous electrolytes. Nanoscale Res. Lett. 2016, 11, 18.

(28) Rivera, L. M.; García, G.; Pastor, E. Novel graphene materials for the oxygen reduction reaction. Curr. Opin. Electrochem. 2018, 9, 233.

(29) Iglesias, D.; Giuliani, A.; Melchionna, M.; Marchesan, S.; Criado, A.; Nasi, L.; Bevilacqua, M.; Tavagnacco, C.; Vizza, F.; Prato, M.; Fornasiero, P. N-doped graphitized carbon nanohorns as a forefront electrocatalyst in highly selective $\mathrm{O}_{2}$ reduction to $\mathrm{H}_{2} \mathrm{O}_{2}$. Chem 2018, 4, 106.

(30) Kim, H. W.; Ross, M. B.; Kornienko, N.; Zhang, L.; Guo, J.; Yang, P.; McCloskey, B. D. Efficient hydrogen peroxide generation using reduced graphene oxide-based oxygen reduction electrocatalysts. Nat. Catal. 2018, 1, 282.

(31) Lu, Z.; Chen, G.; Siahrostami, S.; Chen, Z.; Liu, K.; Xie, J.; Liao, L.; Wu, T.; Lin, D.; Liu, Y.; Jaramillo, T. F.; Nørskov, J. K.; Cui, $\mathrm{Y}$. High-efficiency oxygen reduction to hydrogen peroxide catalysed by oxidized carbon materials. Nat. Catal. 2018, 1, 156.

(32) Banks, C. E.; Davies, T. J.; Wildgoose, G. G.; Compton, R. G. Electrocatalysis at graphite and carbon nanotube modified electrodes: edge-plane sites and tube ends are the reactive sites. Chem. Commun. 2005, 0, 829.

(33) Wu, L.; Zhang, X.; Ju, H. Detection of NADH and ethanol based on catalytic activity of soluble carbon nanofiber with low overpotential. Anal. Chem. 2007, 79, 453.

(34) Zhou, M.; Guo, J.; Guo, L.-p.; Bai, J. Electrochemical sensing platform based on the highly ordered mesoporous carbon-fullerene system. Anal. Chem. 2008, 80, 4642.

(35) Rabti, A.; Raouafi, N.; Merkoçi, A. Bio(sensing) devices based on ferrocene-functionalized graphene and carbon nanotubes. Carbon 2016, 108, 481

(36) Wang, J.; Musameh, M.; Lin, Y. Solubilization of carbon nanotubes by nafion toward the preparation of amperometric biosensors. J. Am. Chem. Soc. 2003, 125, 2408.

(37) Wang, J.; Musameh, M. Carbon nanotube/teflon composite electrochemical sensors and biosensors. Anal. Chem. 2003, 75, 2075.

(38) Pumera, M.; Merkoçi, A.; Alegret, S. Carbon nanotube-epoxy composites for electrochemical sensing. Sens. Actuators, B 2006, 113, 617.

(39) Wang, J.; Yang, S.; Guo, D.; Yu, P.; Li, D.; Ye, J.; Mao, L. Comparative studies on electrochemical activity of graphene nanosheets and carbon nanotubes. Electrochem. Commun. 2009, 11, 1892.

(40) Takahashi, S.; Abiko, N.; Anzai, J.-I. Redox response of reduced graphene oxide-modified glassy carbon electrodes to hydrogen peroxide and hydrazine. Materials 2013, 6, 1840.

(41) Zhou, M.; Zhai, Y.; Dong, S. Electrochemical sensing and biosensing platform based on chemically reduced graphene oxide. Anal. Chem. 2009, 81, 5603.

(42) Fan, L.; Zhang, Q.; Wang, K.; Li, F.; Niu, L. Ferrocene functionalized graphene: preparation, characterization and efficient electron transfer toward sensors of $\mathrm{H}_{2} \mathrm{O}_{2}$. J. Mater. Chem. 2012, 22, 6165.

(43) Zhang, R.; Chen, W. Recent advances in graphene-based nanomaterials for fabricating electrochemical hydrogen peroxide sensors. Biosens. Bioelectron. 2017, 89, 249.

(44) Hamilton, C. E.; Lomeda, J. R.; Sun, Z.; Tour, J. M.; Barron, A. R. High-yield organic dispersions of unfunctionalized graphene. Nano Lett. 2009, 9, 3460. 
(45) Liang, Y.; Wu, D.; Feng, X.; Müllen, K. Dispersion of graphene sheets in organic solvent supported by ionic interactions. Adv. Mater. 2009, 21, 1679.

(46) Woo, S.; Kim, Y.-R.; Chung, T. D.; Piao, Y.; Kim, H. Synthesis of a graphene-carbon nanotube composite and its electrochemical sensing of hydrogen peroxide. Electrochim. Acta 2012, 59, 509.

(47) Wang, Q.; Li, M.; Szunerits, S.; Boukherroub, R. Environmentally friendly reduction of graphene oxide using tyrosine for nonenzymatic amperometric $\mathrm{H}_{2} \mathrm{O}_{2}$ detection. Electroanalysis 2014, 26, 156.

(48) Yeh, M.-H.; Li, Y.-S.; Chen, G.-L.; Lin, L.-Y.; Li, T.-J.; Chuang, H.-M.; Hsieh, C.-Y.; Lo, S.-C.; Chiang, W.-H.; Ho, K.-C. Facile synthesis of boron-doped graphene nanosheets with hierarchical microstructure at atmosphere pressure for metal-free electrochemical detection of hydrogen peroxide. Electrochim. Acta 2015, 172, 52.

(49) Eng, A. Y. S.; Chua, C. K.; Pumera, M. Refinements to the structure of graphite oxide: absolute quantification of functional groups via selective labelling. Nanoscale 2015, 7, 20256.

(50) Liao, L.; Peng, H.; Liu, Z. Chemistry makes graphene beyond graphene. J. Am. Chem. Soc. 2014, 136, 12194.

(51) Park, J.; Yan, M. Covalent functionalization of graphene with reactive intermediates. Acc. Chem. Res. 2013, 46, 181.

(52) Poulos, T. L.; Kraut, J. The stereochemistry of peroxidase catalysis. J. Biol. Chem. 1980, 255, 8199.

(53) Bakandritsos, A.; Pykal, M.; Błoński, P.; Jakubec, P.; Chronopoulos, D. D.; Poláková, K.; Georgakilas, V.; Čépe, K.; Tomanec, O.; Ranc, V.; Bourlinos, A. B.; Zbořil, R.; Otyepka, M. Cyanographene and graphene acid: emerging derivatives enabling high-Yield and selective functionalization of graphene. ACS Nano 2017, 11, 2982.

(54) Radhakrishnana, S.; Kim, S. J. An enzymatic biosensor for hydrogen peroxide based on one-pot preparation of $\mathrm{CeO}_{2}$-reduced graphene oxide nanocomposite. RSC Adv. 2015, 5, 12937.

(55) Mayo, D. W. in Course Notes on the interpretation of infrared and raman spectra; Mayo, D. W.; Miller, F. A.; Hannah, R. W. Ed., John Wiley \& Sons: Hoboken, New Jersey 2003; pp. 101-140.

(56) Szabó, T.; Berkesi, O.; Forgó, P.; Josepovits, K.; Sanakis, Y.; Petridis, D.; Dékány, I. Evolution of surface functional groups in a series of progressively oxidized graphite oxides. Chem. Mater. 2006, $18,2740$.

(57) Jha, S. K.; Vasiliev, I. Vibrational signatures of carboxylated graphene: a first-principles study. J. Phys. Chem. C 2018, 122, 24996.

(58) Eda, G.; Fanchini, G.; Chhowalla, M. Large-area ultrathin films of reduced graphene oxide as a transparent and flexible electronic material. Nat. Nanotechnol. 2008, 3, 270.

(59) Gupta, B.; Kumar, N.; Panda, K.; Kanan, V.; Joshi, S.; VisolyFisher, I. Role of oxygen functional groups in reduced graphene oxide for lubrication. Sci. Rep. 2017, 7, 45030.

(60) Chen, X.; Wu, G.; Cai, Z.; Oyama, M.; Chen, X. Advances in enzyme-free electrochemical sensors for hydrogen peroxide, glucose, and uric acid. Microchim. Acta 2014, 181, 689.

(61) Cheong, Y. H.; Nasir, M. Z. M.; Bakandritsos, A.; Pykal, M.; Jakubec, P.; Zboril, R.; Otyepka, M.; Pumera, M. Cyanographene and graphene acid: the functional group of graphene derivative determines the application in electrochemical sensing and capacitors. ChemElectroChem 2019, 6, 229.

(62) Chai, J.-D.; Head-Gordon, M. Long-range corrected hybrid density functionals with damped atom-atom dispersion corrections. Phys. Chem. Chem. Phys. 2008, 10, 6615.

(63) Dunning, T. H., Jr. Gaussian basis sets for use in correlated molecular calculations. I. The atoms boron through neon and hydrogen. J. Chem. Phys. 1989, 90, 1007.

(64) Marenich, A. V.; Cramer, C. J.; Truhlar, D. G. Universal solvation model based on solute electron density and on a continuum model of the solvent defined by the bulk dielectric constant and atomic surface tensions. J. Phys. Chem. B 2009, 113, 6378. 